\title{
ARTICLE OPEN Deep learning enables robust assessment and selection of human blastocysts after in vitro fertilization
}

\author{
Pegah Khosravi ${ }^{1,2}$, Ehsan Kazemi ${ }^{3}$, Qiansheng Zhan ${ }^{4}$, Jonas E. Malmsten (iD ${ }^{4}$, Marco Toschi ${ }^{4}$, Pantelis Zisimopoulos ${ }^{1,2}$, \\ Alexandros Sigaras ${ }^{1,2}$, Stuart Lavery ${ }^{5}$, Lee A. D. Cooper (iD $^{6}$, Cristina Hickman ${ }^{5}$, Marcos Meseguer ${ }^{7}$, Zev Rosenwaks ${ }^{4}$, \\ Olivier Elemento ${ }^{1,2,8}$, Nikica Zaninovic ${ }^{4}$ and Iman Hajirasouliha ${ }^{1,2}$
}

Visual morphology assessment is routinely used for evaluating of embryo quality and selecting human blastocysts for transfer after in vitro fertilization (IVF). However, the assessment produces different results between embryologists and as a result, the success rate of IVF remains low. To overcome uncertainties in embryo quality, multiple embryos are often implanted resulting in undesired multiple pregnancies and complications. Unlike in other imaging fields, human embryology and IVF have not yet leveraged artificial intelligence (Al) for unbiased, automated embryo assessment. We postulated that an Al approach trained on thousands of embryos can reliably predict embryo quality without human intervention. We implemented an Al approach based on deep neural networks (DNNs) to select highest quality embryos using a large collection of human embryo time-lapse images (about 50,000 images) from a high-volume fertility center in the United States. We developed a framework (STORK) based on Google's Inception model. STORK predicts blastocyst quality with an AUC of $>0.98$ and generalizes well to images from other clinics outside the US and outperforms individual embryologists. Using clinical data for 2182 embryos, we created a decision tree to integrate embryo quality and patient age to identify scenarios associated with pregnancy likelihood. Our analysis shows that the chance of pregnancy based on individual embryos varies from $13.8 \%$ (age $\geq 41$ and poor-quality) to $66.3 \%$ (age $<37$ and good-quality) depending on automated blastocyst quality assessment and patient age. In conclusion, our Al-driven approach provides a reproducible way to assess embryo quality and uncovers new, potentially personalized strategies to select embryos.

npj Digital Medicine (2019)2:21 ; https://doi.org/10.1038/s41746-019-0096-y

\section{INTRODUCTION}

Infertility remains an unremitting reproductive issue that affects about 186 million people worldwide. ${ }^{1}$ In the United States, infertility affects $\sim 8 \%$ of women of child-bearing age. ${ }^{2}$

In vitro fertilization (IVF) is one of the most common treatments for infertility. IVF involves ovarian stimulation followed by the retrieval of multiple oocytes, fertilization, and embryo culture for 1-6 days in controlled environmental conditions. Although IVF and embryo-transfer technologies have improved considerably over the past 30 years, the efficacy of IVF remains relatively low. ${ }^{3}$

Conventional embryo evaluation involves manual grading of human embryos at the blastocyst stage (embryo on day 5) based on morphological analysis by skilled embryologists. ${ }^{4}$ While this selection method is used universally in clinical practice, the evaluation of an embryo based on a static image represents a crude, subjective evaluation of embryo quality, which is incomplete as well as time-consuming. ${ }^{5-7}$

Moreover, there continues to be a tendency for inconsistent blastocyst classification, often associated with different grading systems among medical centers. Indeed, attempts to establish a universal grading and selection system have thus far failed to catch on. ${ }^{8}$

Improving the ability to select the single best embryo with the highest implantation potential would increase pregnancy rates as well as minimize the chance of multiple pregnancies due to the transfer of multiple embryos. ${ }^{9}$ Time-lapse imaging $(T L I)^{10}$ is an emerging technology that allows continuous observation of embryo development without removing embryos from controlled and stable incubator conditions. ${ }^{11}$ However, even though TLI represents a step toward more objective embryo evaluation, the inter- and intra-evaluator variation among embryologists using conventional morphological grading and/or TLI annotations is well documented. ${ }^{12-14}$

There are various efficient machine learning methods, which due to their relatively better performances in various fields of research are utilized for embryo classification. Two recent studies have attempted to use some of these approaches for embryo quality analysis, with varying degrees of success ${ }^{15,16}$ on a limited bovine and mammalian oocytes data, using Al- and random forest (RF)-based classification, respectively. Their results showed $76.4 \%$ (test set $=73$ embryos) and $75 \%$ (test

\footnotetext{
${ }^{1}$ Institute for Computational Biomedicine, Department of Physiology and Biophysics, Weill Cornell Medicine of Cornell University, New York, NY, USA; ${ }^{2}$ Caryl and Israel Englander Institute for Precision Medicine, The Meyer Cancer Center, Weill Cornell Medicine, New York, NY, USA; ${ }^{3}$ Yale Institute for Network Science, Yale University, New Haven, CT, USA; ${ }^{4}$ The Ronald O. Perelman and Claudia Cohen Center for Reproductive Medicine, Weill Cornell Medicine, New York, NY, USA; ${ }^{5}$ Institute of Reproduction and Developmental

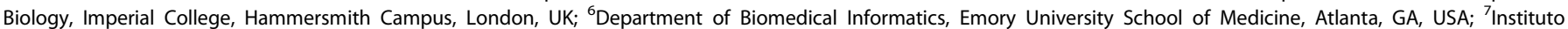
Valenciano de Infertilidad, Universidad de Valencia, Valencia, Spain and ${ }^{8}$ WorldQuant Initiative for Quantitative Prediction, Weill Cornell Medicine, New York, NY, USA Correspondence: Nikica Zaninovic (nizanin@med.cornell.edu) or Iman Hajirasouliha (imh2003@med.cornell.edu)

These authors contributed equally: Zev Rosenwaks, Olivier Elemento, Nikica Zaninovic, Iman Hajirasouliha
} 
set $=56$ embryos) accuracy for discretization of bovine embryo grades and mammalian oocyte grades, respectively. Furthermore, a few previously published approaches have focused on classifying human blastocyst quality based on specific features, such as the inner cell mass (ICM) area, trophectoderm (TE) area, zona pellucida (ZP) thickness, and blastocyst area and radius separately. ${ }^{9,17}$ In particular, Filho et al. ${ }^{17}$ presented a semiautomatic grading system of human embryos. The authors showed that classifiers can have different accuracies for each embryo component (blastocyst extension, ICM, and TE). Their results indicated various accuracy ranges from 67 to $92 \%$ for the embryo extension, from 67 to $82 \%$ for the ICM, and from 53 to 92\% for TE detection; 92\% was the highest accuracy achieved across a 73-embryo test set. ${ }^{17}$ Although these methods achieved reasonable accuracy in assessing human embryo quality, they require advanced embryological expertize and several preprocessing steps, and do not scale to large datasets.

Deep learning algorithms, in particular convolutional neural networks (CNNs), have recently been used to address a number of medical-imaging problems, such as detection of diabetic retinopathy, $^{18}$ skin lesions, $^{19}$ and diagnosing disease. ${ }^{20}$ They have become the technique of choice in computer vision and they are the most successful type of models for image analysis. Unlike regular neural networks, CNNs contain neurons arranged in three dimensions (i.e., width, height, depth). Recently, deep architectures of CNNs such as Inception ${ }^{21}$ and ResNet ${ }^{22}$ have dramatically increased the progress rate of deep learning methods in image classification. ${ }^{23}$ In this paper, we sought to use deep learning to accurately predict the quality of human blastocysts and help select the best single embryo for transfer (Fig. 1).

\section{RESULTS}

Deep neural network achieves a highly accurate classification of embryo images

We used time-lapse images from 10,148 human embryos, obtained from the Center for Reproductive Medicine at Weill Cornell Medicine to train and validate our DNN. The 10,148 embryos (WCM-NY dataset) were classified into three major quality groups, good-quality ( $n=1345$ embryos), fair-quality ( $n=4062$ embryos), and poor-quality ( $n=4741$ embryos) (Fig. $2 \mathrm{a}, \mathrm{b}$ ) based on their assigned grades (see Methods). We obtained time-lapse images from each of the embryos, each consisting of several time points, seven focal depths per time point (Fig. 2a, b) and $500 \times 500$ pixels black and white images per focal depth $(+45$, $+30,+15,0,-15,-30$, and -45). Upon preprocessing and removal of images with readability issues (e.g., those with a dark background) and random selection of a balanced set of images (see Methods), we were left with a total of 12,001 images from up to seven focal depths: 6000 images in 877 good-quality embryos, and 6001 images in 887 poor-quality embryos.

We then trained an Inception-V1 DNN-based algorithm using the two quality groups at both ends of the spectrum, i.e., good-quality and poor-quality. The Inception-V1 architecture is a transfer learning algorithm, where we initially performed fine-tuning of the parameters for all of the layers. We used 50,000 steps for training the DNN and subsequently evaluated the performance of our DNN (called STORK) using a randomly selected independent test set with 964 good-quality images from 141 embryos and 966 poor-quality images from 142 embryos. Our results showed that the trained algorithm was able to identify good-quality and poor-quality images with $96.94 \%$ accuracy (1871 correct predictions out of 1930 images).

To measure the accuracy of STORK for individual embryos, we used a simple voting system across multiple image focal depths. If
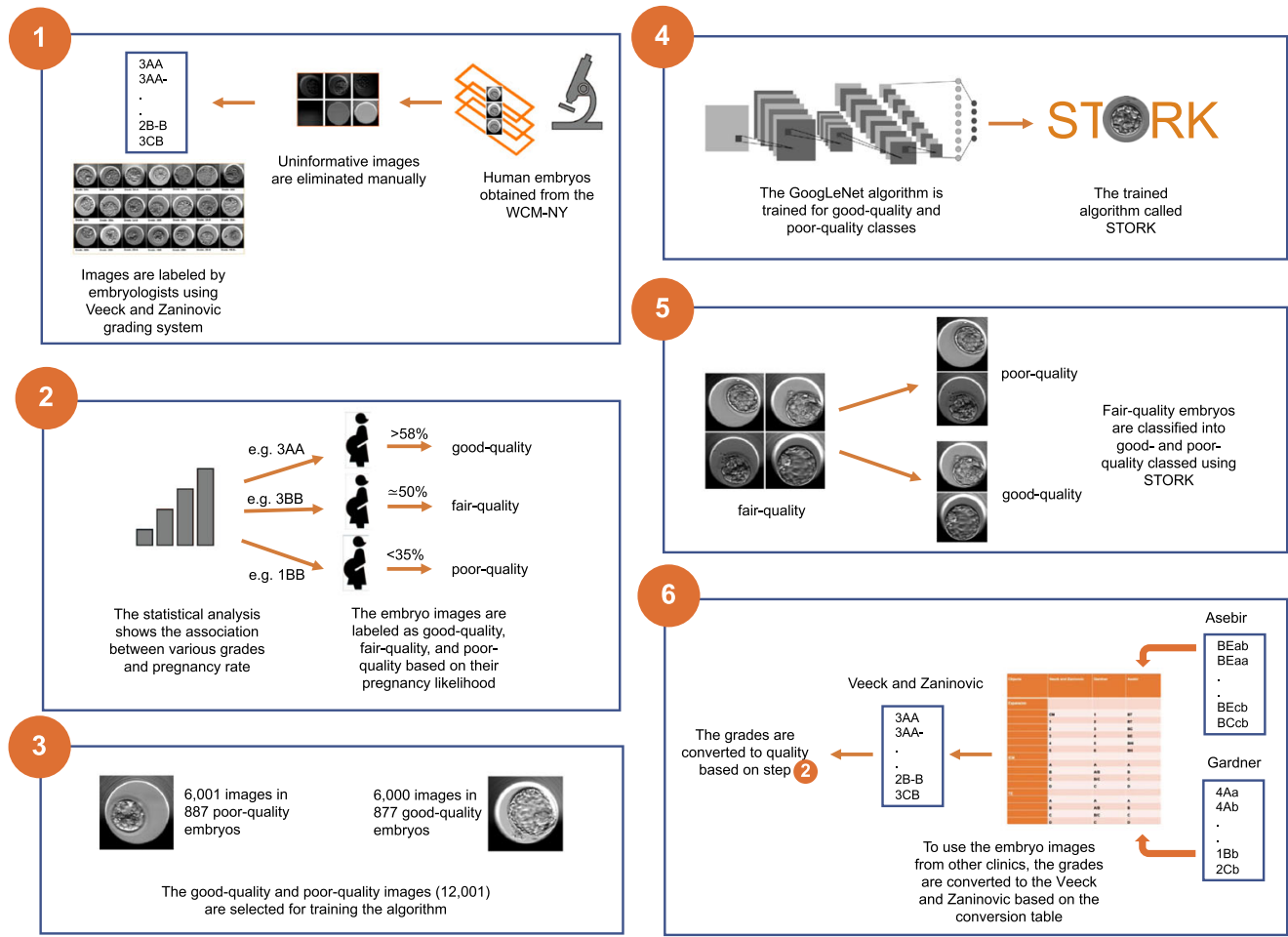
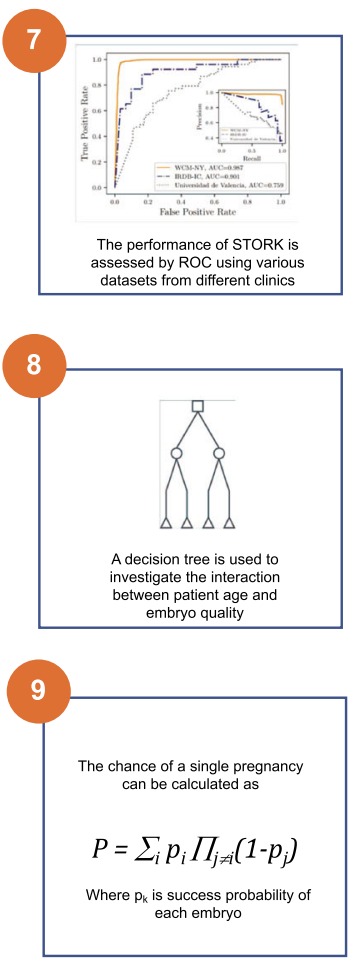

Fig. 1 The STORK flowchart: This flowchart illustrates the design and assessment of STORK. First, Human embryo images are provided from the embryology lab and labeled by embryologists as good-quality or poor-quality based on their pregnancy likelihood. Then, the labels and clinical information from the extracted images are integrated, and the Inception-V1 algorithm is trained for good-quality and poor-quality classes. Furthermore, STORK is evaluated by a blind test set to assess its performance in predicting embryo quality. Finally, the CHAID decision tree is used to investigate the interaction between patient age and embryo quality 
a

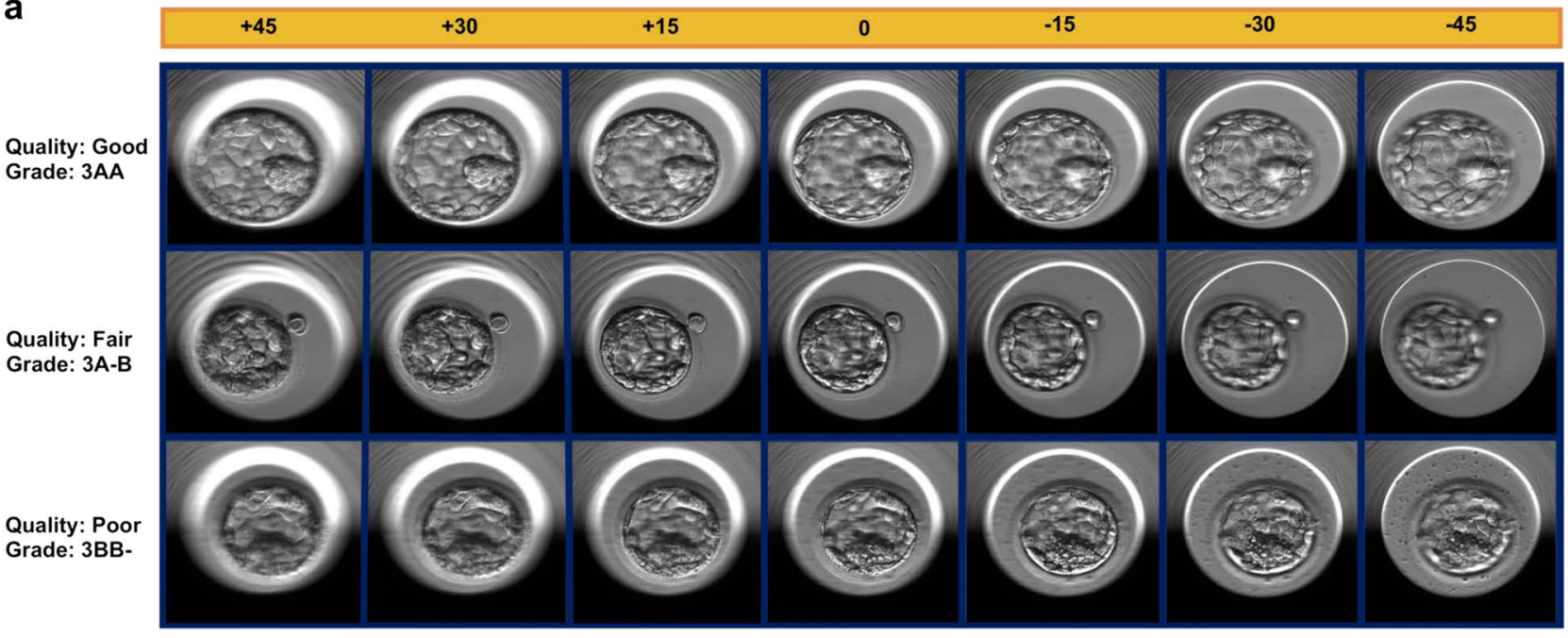

b

Quality: Poor Grade: 3BB

Good-quality
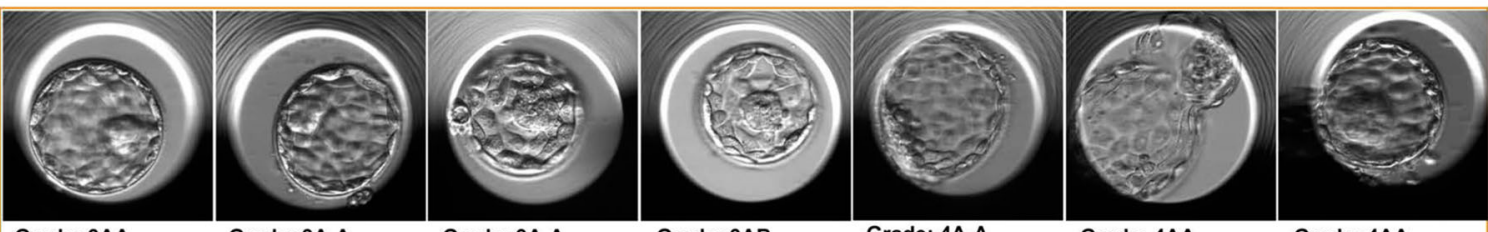

Grade: 3A-A

Grade: 3A-A-

Grade: $3 A B$

Grade: 4A-A

Grade: 4AA-

Grade: 4AA
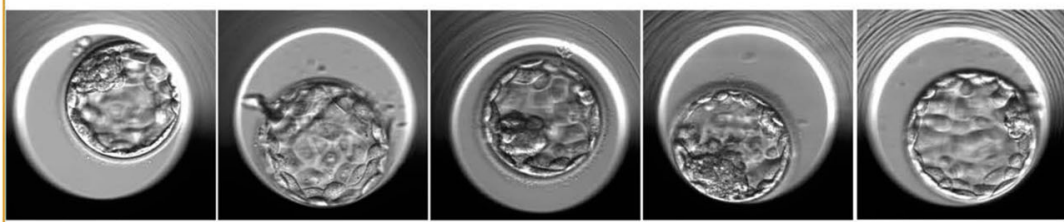

Grade: 3BA-

Grade: 3A-B

Grade: 3BB

Grade: 3BA-
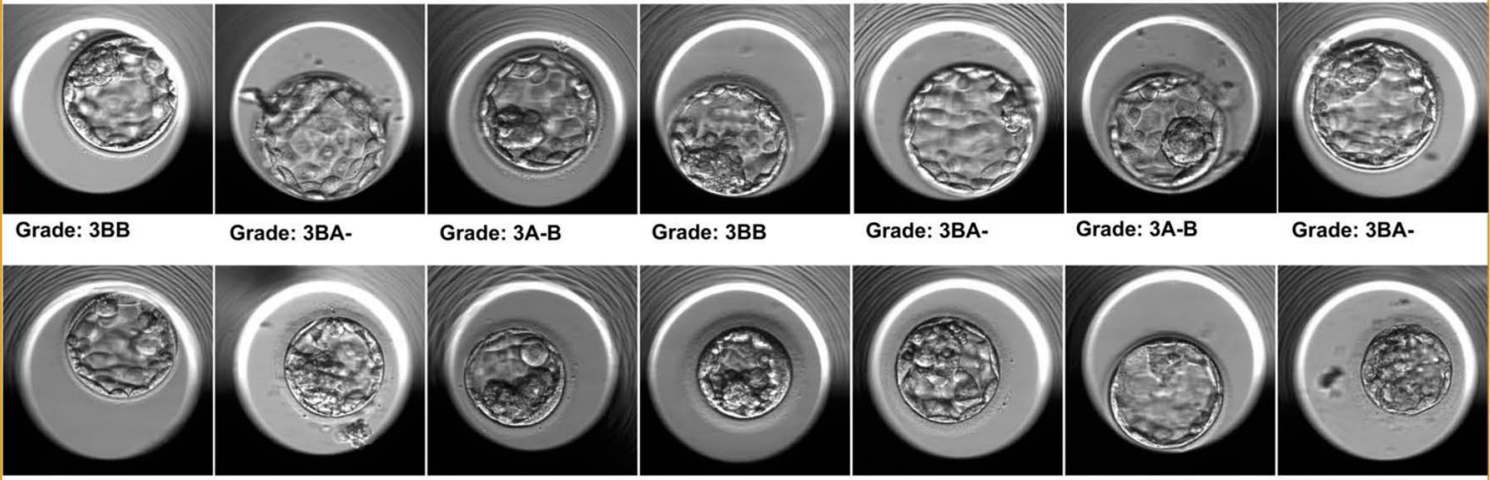

Grade: 3A-B

Grade: 3BA-

Grade: 3BB-

Grade: 2BB

Grade: 2B-B-

Grade: 1BB

Grade: 2BB-

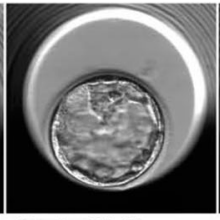

Grade: 3B-B

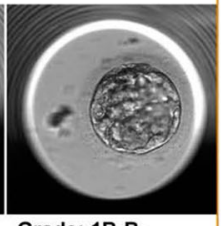

Fig. 2 Embryologists' evaluation: a This figure shows three examples of Veeck and Zaninovich grades and their corresponding quality labels across seven focal depths. b Embryologists evaluate embryo quality using an internal scoring system and subsequently classify them into three major groups (good-quality, fair-quality, poor-quality) based on the pregnancy rate

the majority of images from the same embryo were predicted to be of good-quality, then the final quality of the embryo was considered good. For a small number of cases in which the number of good-quality and poor-quality images was equal (e.g., three good-quality and three poor-quality for six focal depths), we used STORK's output probability scores to break the tie. At the embryo level, we obtained $97.53 \%$ accuracy with 276 correct predictions out of 283 embryos.

At the image level, we observed an average area under the curve (AUC) of 0.987 (Fig. 3a) on the blind test set. We also found that training an Inception-V1 model without parameter finetuning did not affect performance (accuracy; Fig. 3b). This observation is in agreement with previous studies using these deep learning techniques. ${ }^{20,24,25}$

We also found that STORK classified the fair-quality embryo (intermediate group, Figs. 2 and 4) images (4480 images from 640 embryos) as $82 \%$ good-quality (526 embryos) and $18 \%$ poorquality (114 embryos), respectively. As Inception-V1 was trained for good-quality and poor-quality classes with different pregnancy probabilities (an $\sim 58 \%$ and $35 \%$ chance of pregnancy for goodquality and poor-quality classes, respectively), we wondered if STORK nonetheless produced relevant predictions (association between embryo quality and pregnancy rate) within the fairquality class. A closer look showed that embryos with fair-quality images that were classified as poor-quality by STORK had a lower likelihood of positive live birth $(50.9 \%)$ as compared to those classified as good-quality (61.4\% positive live birth; $p<0.05$ by the two-tailed Fisher's test). Note that STORK alone cannot estimate the pregnancy rate. However, it can detect the association between embryo quality and pregnancy rate based on morphological classification.

In addition, we found that fair-quality embryos predicted to be good-quality by STORK came from younger patients (33.9 years old on average) than those predicted to be poor-quality (34.25 years old on average). Interestingly, these numbers are similar to the age of patients with good-quality and poor-quality embryos: 33.86 and 34.72 years old on average, respectively. This suggests 

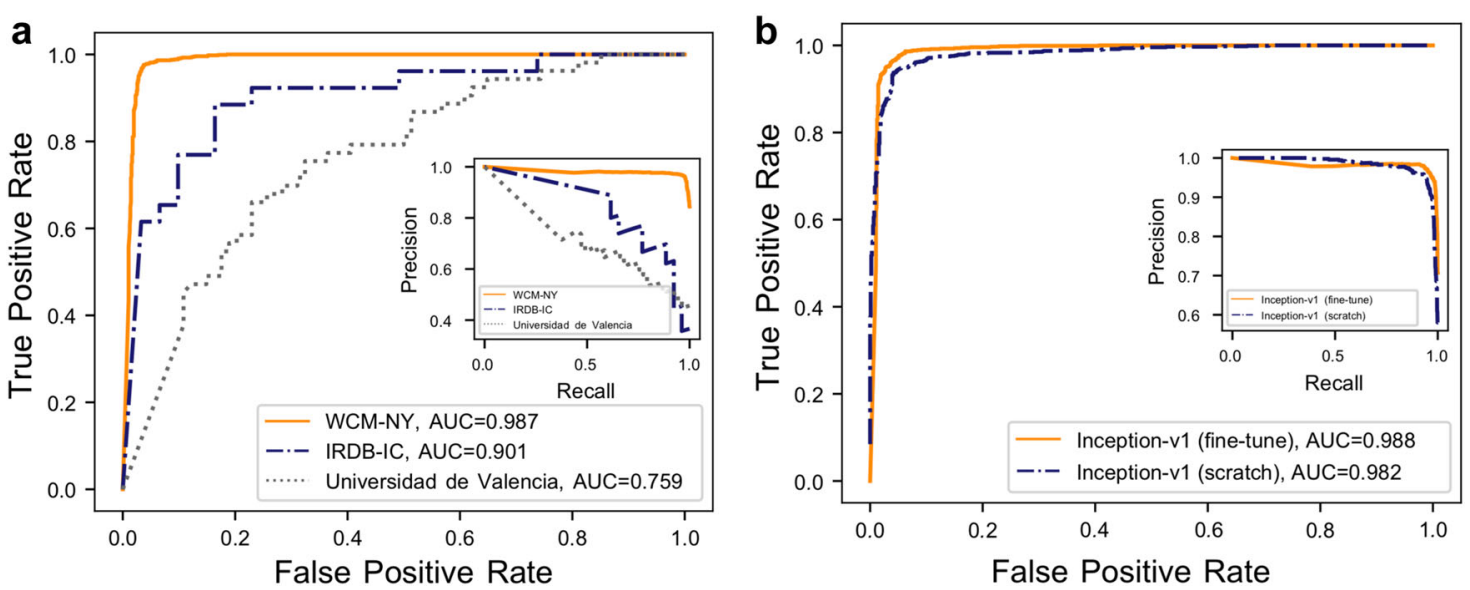

Fig. 3 Deep neural network results: a Inception-V1 (fine-tuning the parameters for all layers) results for three datasets. b Inception-V1 via two different training methods (fine-tuning the parameters for all layers and training from scratch) in good-quality and poor-quality embryo quality discrimination dataset. WCM-NY: data from the Center for Reproductive Medicine and Infertility at Weill Cornell Medicine of New York; IRDB-IC: data from the Institute of Reproduction and Developmental Biology of Imperial College; Universidad de Valencia: data from the Institute Valenciano de Infertilidad, Universidad de Valencia

that STORK finds sufficient structure within embryos classified as fair-quality to make clinically relevant predictions (Fig. 4).

\section{STORK is robust when applied to datasets from other clinics}

To evaluate STORK's robustness, we tested its performance by using additional datasets of embryo images obtained from two other IVF centers, Universidad de Valencia and IRDB-IC, comprising 127 (74 good-quality, 53 poor-quality) and 87 (61 good-quality, 26 poor-quality) embryos, respectively (Supplementary Table 2). Our experimental results (See Fig. 3a) demonstrate that although the scoring systems used for these centers are different from the system used to train our model, STORK can successfully identify and register score variations and robustly discriminate between them, with an AUC of 0.90 and 0.76 for the IRDB-IC and Universidad de Valencia and (Fig. 3a), respectively. Lower concordance of the classification results (by STORK) for the Universidad de Valencia dataset could be related to different grading systems used by that clinic. The images of Universidad de Valencia dataset are labeled using Asebir ${ }^{26}$ while IRDB-IC is labeled using the Gardner system. ${ }^{27}$ The Veeck and Zaninovic grading system is a slightly modified version of the Gardner system (Supplementary Table 4).

STORK outperforms individual embryologists for embryo selection It is well known that embryo scoring frequently varies among embryologists, ${ }^{28}$ mainly due to the subjectivity of the scoring process and different interpretations of embryo quality. We, therefore, sought to create a small but robust benchmark embryo dataset that would represent the consensus of several embryologists. We asked five embryologists from three different clinics to provide scores for each of 394 embryos generated in different labs (Supplementary Table 6). Note that these images were not used in the training phase of our algorithm. The embryo images were scored using the Gardner scoring system ${ }^{27}$ and then mapped onto our simplified three groups (good-quality, fair-quality, and poorquality; see Supplementary Table 4 for the mapping method).

As expected, we found a low level of agreement among the embryologists (Supplementary Fig. 1b), with only 89 embryos out of the 394 classified as the same quality by all five embryologists (Supplementary Fig. 1a). Therefore, to create a larger and more accurate gold standard dataset, we used an embryologist majority voting procedure (i.e., the quality of each image was determined by the score given by at least three out of the five embryologists) to classify 239 images (32 good-quality and 207 poor-quality).
When we applied STORK to these 239 images, we found that it predicted the embryologist majority vote with precision of $95.7 \%$ (Cohen's kappa $=0.63$ ). In comparison, STORK agreed with each individual embryologist as follows: $0.69,0.54,0.25,0.62$, and 0.54 Cohen's kappa score. These results indicate that STORK may outperform individual embryologists when assessing embryo image quality (Fig. 5).

A decision tree predicts likelihood of successful pregnancy based on embryo quality and clinical parameters

It is known that other factors, besides embryo quality, such as patient age, the patient's genetic background, clinical diagnosis, and treatment-related characteristics, can affect pregnancy outcome. ${ }^{29,30}$ As embryo quality is one of the most important of these factors, the ultimate aim of any embryo assessment approach is to identify embryos that have the highest implantation potential resulting in live birth. ${ }^{27,31,32}$ However, embryo quality alone is not enough to accurately determine the pregnancy probability (see Supplementary Method 1, Supplementary Method 2 and Supplementary Fig. 2).

Therefore, in this section we present an alternative method for predicting successful pregnancy probability based on a state-ofthe-art decision tree method that integrates clinical information and embryo quality. We wondered if we could assess the successful pregnancy rate by using a combination of embryo quality and patient age, as age is one of the most important clinical variables. For this purpose, we used a hierarchical decision tree method known as chi-squared automatic interaction detection (CHAID) algorithm. ${ }^{33}$

We designed a CHAID ${ }^{34,35}$ decision tree using 2182 embryos from the WCM-NY database (Supplementary Table 6) with available clinical information and pregnancy outcome results (Fig. 6). We then investigated the interaction between patient age (consisting of seven classes: $\leq 30,31-32,33-34,35-36,37-38$, $39-40$, and $\geq 41$ ) (Supplementary Fig. 3a) and embryo quality (consisting of two classes: good-quality and poor-quality). The fully de-identified data consists of a very diverse population of patients (Supplementary Fig. 3b). The effect on live birth outcome is demonstrated in (Supplementary Fig. 3c). The CHAID algorithm can project interactions between variables and non-linear effects, which are generally missed by traditional statistical techniques. CHAID builds a tree to determine how variables can explain an outcome in a statistically meaningful way. ${ }^{34,35}$ CHAID uses chisquared statistics for identification of optimal multi-way splits, and 

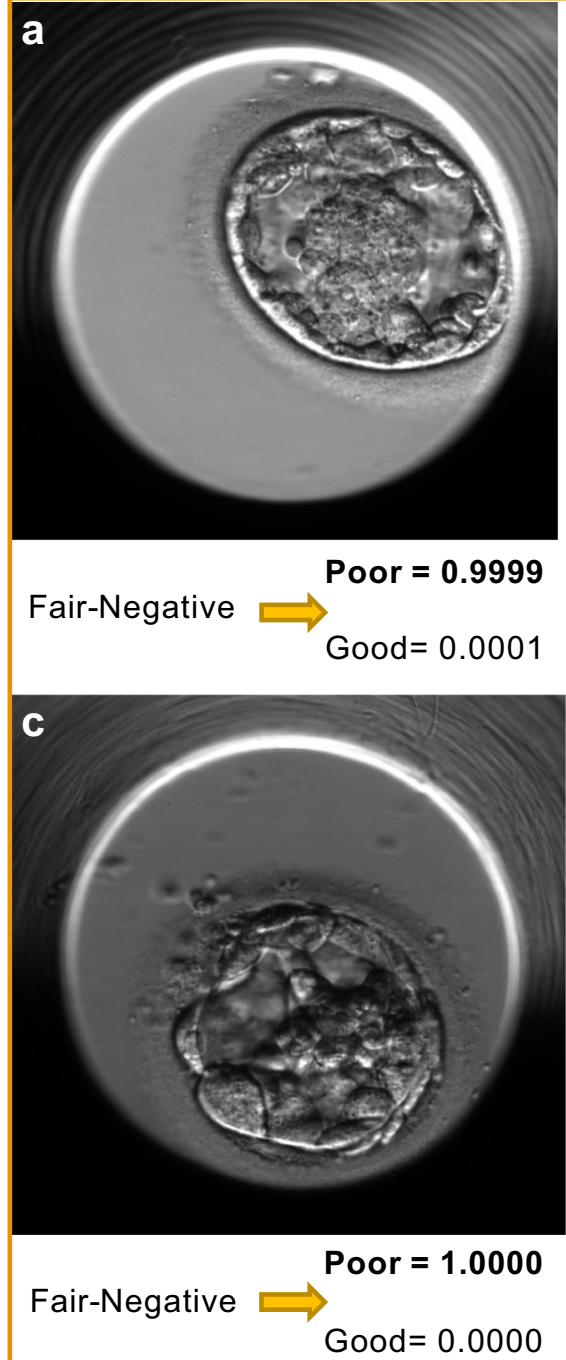
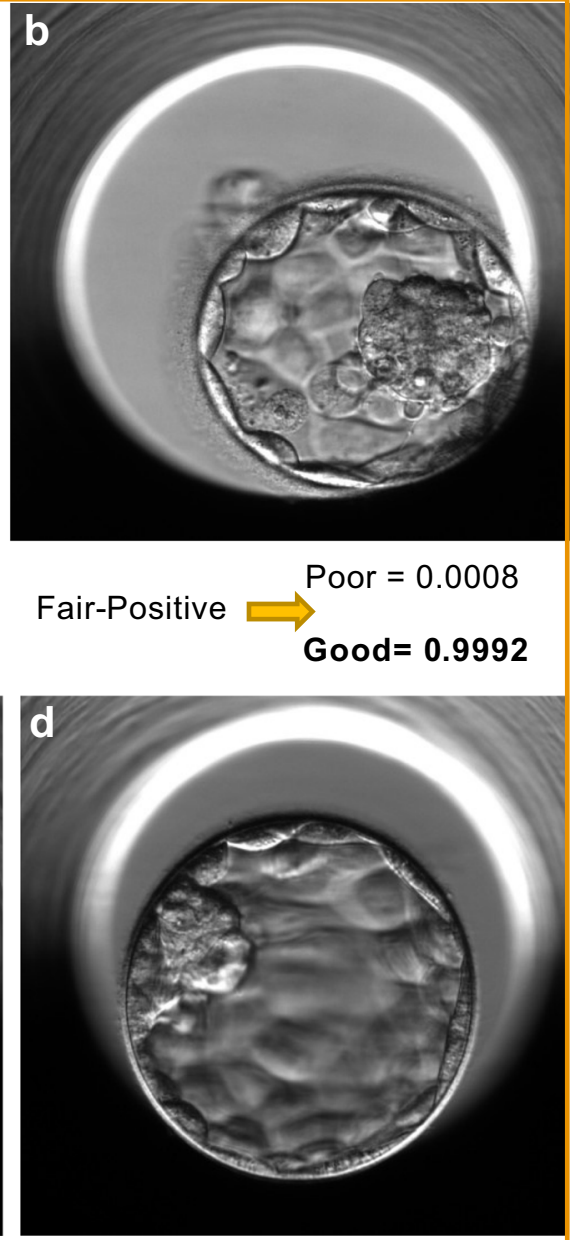

Fair-Positive

Good $=1.0000$

Fig. 4 STORK vs. embryologists classification: STORK classifies the fair-quality images into existing good-quality and poor-quality classes. For example, panels "a" and "b" are labeled 3A-B (fair-quality) according to the Veeck and Zaninovic grading system, while STORK classified them as poor-quality and good-quality, respectively. Also, panels "c" and "d" are both labeled 3BB (fair-quality). However, the algorithm correctly classified panel "c" as poor-quality and panel "d" as good-quality. As the figure shows, the outcome in the embryos in "b" and "d" is positive live birth, whereas it is negative live birth in "a" and "c"

identifies a set of characteristics (e.g., patient age and embryo quality) that best differentiates individuals based on a categorical outcome (here, live birth) and creates exhaustive and mutually exclusive subgroups of individuals. It chooses the best partition on the basis of statistical significance and uses Bonferroni-adjusted $p$ values to determine significance with a predetermined minimum size of end nodes. We used a 1\% Bonferroni-adjusted $p$-value, a maximum depth of the tree $(n=5)$, and a minimum size of end nodes $(n=20)$ as the stopping criteria. The application of a treebased algorithm on the embryo data would help to more precisely define the effect of patient age and embryo quality (good-quality or poor-quality) on live birth outcome, and to better understand any interactions between these two clinical variables (patient age and embryo quality).

Note that while several other classification algorithms could have been employed for the prediction, CHAID enabled a userfriendly visualization of the resulting decision tree. ${ }^{36,37}$

As Fig. 6 shows, patients were automatically classified into three age groups: (i) $\leq 36$, (ii) 37 and 38 , and (iii) $\geq 39$ years old due to age data distribution. For each age group, embryos were classified in good- and poor-quality groups (Supplementary Fig. 3c).
The results confirm the association between probability of successful pregnancy and patient age. The live birth probability for patients with good-quality embryos is significantly $(1 \%$ Bonferroni-adjusted $p$-value) higher than that for patients with poor-quality embryos across different ages. Figure 6 indicates that patients $\leq 36$ years old have a higher successful pregnancy rate compared to patients in the other two age groups. The CHAID decision tree analysis also indicates that the chance of favorable outcome using IVF varies from $13.8 \%$ (e.g., when the embryo is of poor-quality as assessed by STORK and the patient is $\geq 41$ years old) to $66.3 \%$ (e.g., when the embryo is of good-quality and the patient is $<37$ years old) (Fig. 6).

Probability analysis optimizes embryo selection and maximizes likelihood of single pregnancy

It is a common practice in IVF clinics to select and transfer more than one embryo in order to increase the chance of a successful pregnancy. As the success rate of individual embryos are typically $<50 \%$ and transferring two or more embryos can increase the success probability. However, when the number of transferred 


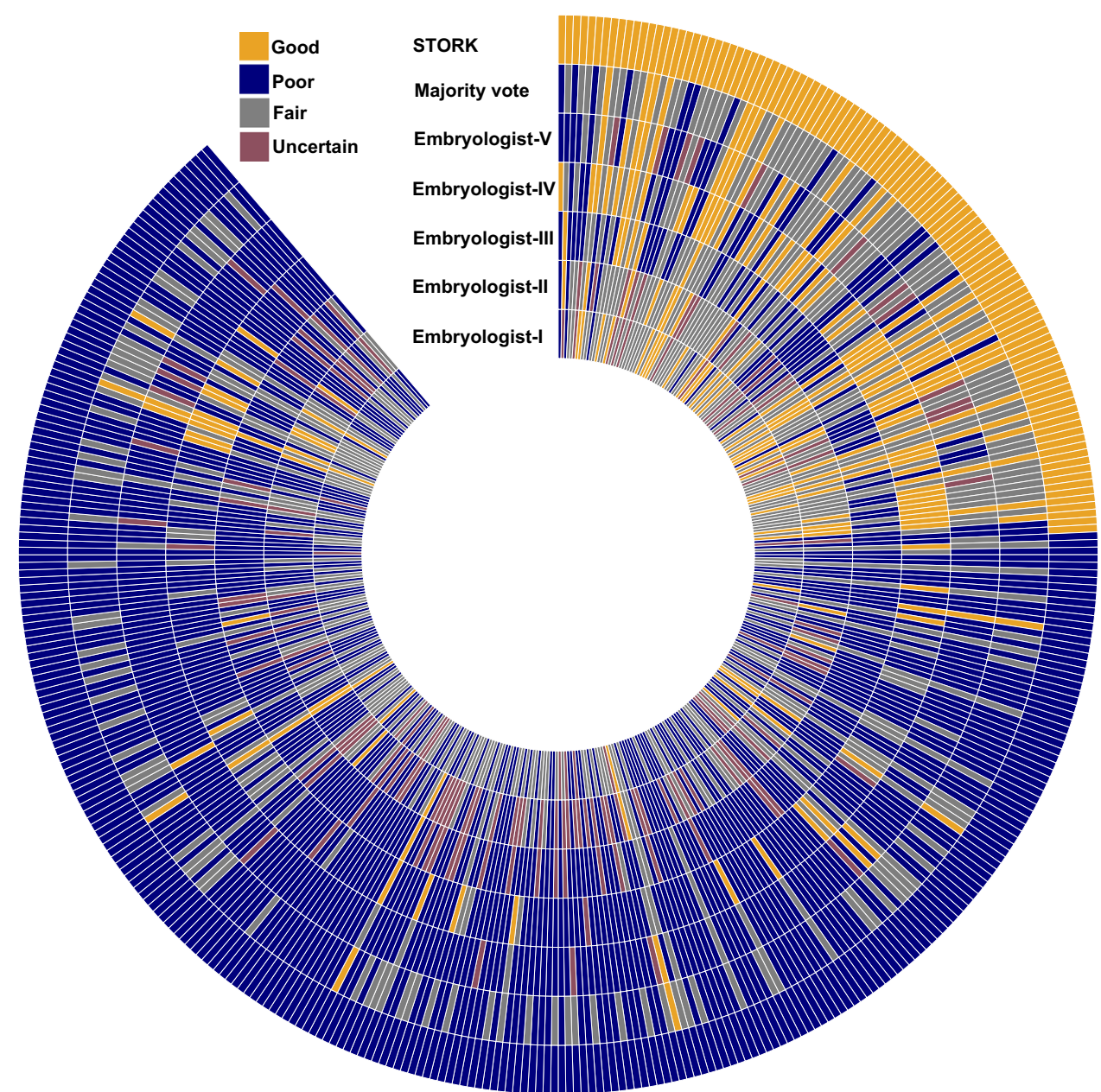

Fig. 5 Assessment comparison of STORK with five embryologists: This circular heatmap demonstrates the prediction of STORK and five embryologists in the labeling of the same images from 394 embryos. STORK outputs good and poor grades. The heatmap compares STORK's result with the majority vote results from all of the embryologists for 239 embryos in which the majority (i.e., at least three out of five embryologists) gives good or poor. The embryologists assess the embryos quality using Gardner grading system. Then, they convert the grades to the three different quality scores as good-quality (orange), fair-quality (gray), and poor-quality (navy) based on the pregnancy rate. Also, for a few embryos, the embryologist uses "?" signs (e.g. 3A?), which refer to the low certainty (red) as they are not sure about the exact label. The heatmap illustrates the result of STORK, Majority vote, Embryologist-V, Embryologist-IV, Embryologist-III, Embryologist-II, and Embryologist-I from the outer circle to the inner ones. Orange: embryos with good-quality; navy: embryos with poor-quality; gray: embryos with fair-quality; red: embryos that are not labeled due to uncertainty

embryos increases, the chance of multiple pregnancies (twins or even triplets) and associated complications also increase. For example, let's simply assume we transfer three embryos each with an independent success probability of $1 / 4$. The chance of a pregnancy can be, thus, calculated as $p=1-(3 / 4)^{3} \approx 0.58$. However, in this scenario, the chance of twin and triplets pregnancy would be $3 \times(1 / 4)^{2}(3 / 4) \approx 0.14$ and $(1 / 4)^{3} \approx 0.02$, respectively. The chance of a single pregnancy would be $3 \times(1 / 4)(3 / 4)^{2} \approx 0.42$.

Given a list of potential embryos for transfer and their predicted success rate using our decision tree analysis, we can calculate the probability of no pregnancy, single pregnancy, and multiple pregnancies, for any selection of embryos from the list. In general, if $k$ embryos are transferred with indicated success probabilities of $p_{1}, \ldots, p_{k}$ (where $p_{i}$ shows the success probability of embryo $i$, for any index $i$ between 1 and $k$ ) then the chance of a single pregnancy can be calculated as $P=\Sigma_{i} p_{i} \Pi_{j \neq i}\left(1-p_{j}\right)$. Given the success rate of any individual embryo transfer, we showed how to calculate the probability of a single successful pregnancy when $k$ embryos $(k>1)$ are transferred. This will help embryologists to select those embryos (for example two or three), which maximize the chance of a single pregnancy when transferred together.

\section{DISCUSSION}

Studies on human embryo evaluation are still very limited and mostly based on morphological features. They often involve low numbers of embryos from single centers, and they lack validations in independent cohorts. Furthermore, publications to date have relied on single static images. However, time-lapse images have the advantage of being consistent in terms of size, lighting, contrast, quality, and in terms of capturing the timing of embryo development, which is particularly important when quantifying blastocyst expansion. Currently, no robust and fully automatic method exists to analyze human embryo data by TLI.

Recently, there have been several studies utilizing classical machine learning approaches, such as support vector machine (SVM) and RF, and deep learning methods, such as CNNbasic, $^{15,16,38}$ for outcome prediction or grade classification. To date, several Al methods have been used to assess blastocysts. ${ }^{39}$ Image segmentation and advanced image analysis techniques using neural networks with textured descriptors, level set, phase congruency, and fitting of ellipse methods have been demonstrated in mouse, ${ }^{40}$ bovine, $^{15}$ and human blastocysts. ${ }^{4,17}$ 


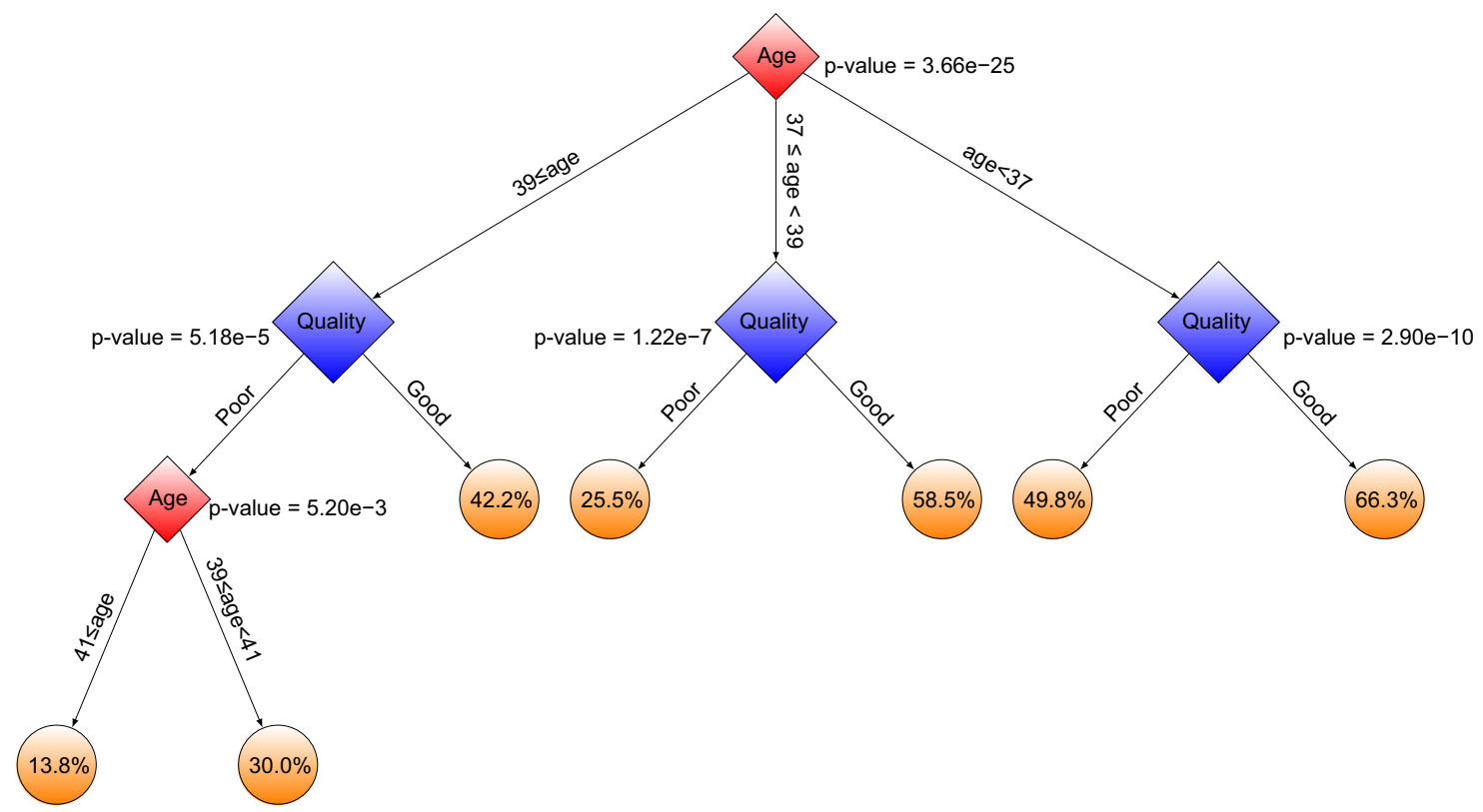

Fig. 6 Interactions between age and embryo quality: The decision tree shows the interactions between IVF patient age and embryo quality using CHAID

More recently, Segal et al. ${ }^{41}$ have developed a random forest classifier-based tool using 2744 embryos to predict which patients should have extended culture with an accuracy of $76.4 \%$. Also, Matsumoto et al. ${ }^{42}$ used time-lapse monitoring of 118 human embryos to determine good-quality embryos using deep learningbased method, which is based on the Keras neural network library. They achieved 70 and $80 \%$ accuracy for the validation dataset through two different cell stages that are significantly lower than the performance obtained from our framework. Besides, the advantage of our method is that instead of only focusing on the predetermined, segmented features that embryologists are trained to analyze, the entire image of the embryo is assessed, allowing for quantification of all the available data. Convolution, therefore, allows the Al to identify patterns in morphological features that we do not know how to assess.

We have demonstrated that deep learning approaches can provide accurate quality assessments in various clinical conditions. The STORK framework presented here provides a method that can be easily implemented for a wide range of applications, including embryo grading. Our method yields a cutting-edge sensitivity when performing the challenging task of assessing embryo quality using multi-focal embryo images. Notably, our STORK framework is fully automated and does not require any manual augmentations or preprocessing on the input images. In fact, it provides embryologists a straightforward platform to use without requiring sophisticated computational knowledge. Furthermore, we designed a decision tree based on the CHAID algorithm to investigate the interaction between embryo quality and patient characteristics (i.e., patient age) in a diverse population, and their effect on the likelihood of live birth.

Finally, although STORK can run on traditional computer microprocessors (CPUs), abundant system memory and graphics processing units (GPUs) make the training process faster (at least one order of magnitude) as training requires loading a significant number of medical images for training and validation.

Nevertheless, our method still has limitations. For example, we explored the possibility of directly predicting the likelihood of pregnancy based on only embryo images that are labeled as "positive live birth" or "negative live birth" (Supplementary Method 2). The result showed that the trained algorithm cannot identify positive live birth and negative live birth successfully using embryo morphology alone.

\section{METHODS}

Images from human blastocysts

This study included 10,148 embryos from the Center for Reproductive Medicine at Weill Cornell Medicine (2012/05-2017/12). We referred to this dataset as WCM-NY throughout this manuscript. This study used retrospective and fully de-identified data. The study was performed in accordance with relevant guidelines and regulations and was approved by the Institutional Review Board at Weill Cornell Medicine (IRB number: 1401014735). The images were captured using the EmbryoScope ${ }^{\circledR}$ timelapse system (Vitrolife, Sweden) with a built-in microscope. Images were captured using single red LED $(635 \mathrm{~nm})$ every $20 \mathrm{~min}$., with seven focal depths $(+45,+30,+15,0,-15,-30$, and -45$)$ of the embryo taken each time, representing a total of 50,392 images $(500 \times 500$ pixels) captured precisely $110 \mathrm{~h}$ post-insemination (hpi) (Fig. 2a). The standardization of images by the EmbryoScope software was consistent, and the images of the blastocysts were labeled using the Veeck and Zaninovic grading system $^{43}$ (Supplementary Table 1). For validation, we also used two external datasets from the Universidad de Valencia, Valencia, Spain, and the Institute of Reproduction and Developmental Biology of Imperial College, London, UK (IRDB-IC) (Supplementary Table 2).

\section{Dataset preparation and the comprised images}

This study presents a framework to classify different embryo images based on blastocyst grading and map those grades to good- and poor-quality.

In machine learning, especially for classification, a high-quality training dataset is important for training the classification model. Therefore, for the first step of selecting images, we manually eliminated the images with dark background and uninformative images (Supplementary Fig. 4) from the training set. Moreover, the number of images with good-quality usually is much less than that of poor-quality within the training sample. To avoid unbalanced classes for training the algorithm, we randomly deleted images from the class, which has sufficient observations (poor-quality) so that the comparative ratio of two classes is equal in our data.

For the next step, the embryologists labeled the embryo images with quality labels (good-quality, fair-quality, and poor-quality) to map certain quantitative scores from the grading system of Veeck and Zaninovic (e.g., $3 \mathrm{AA}, 3 \mathrm{BB}, 1 \mathrm{BB})$ to three quality grades based on the pregnancy outcome obtained from statistical analysis of clinical data. In this regard, any grade that contained B- or C and an extension rate equal to or less than three was considered part of the poor-quality group with $<35 \%$ pregnancy 
chance. In addition, any score with two A or A- grades, or one A with B, with an extension of 3 or greater could be labeled as good-quality with $>58 \%$ pregnancy chance. However, the experts debated about some scores (e.g., 3BB, 3BA-), putting them in a separate category (fair-quality) as their pregnancy likelihood was about $50 \%$. Then, fair-quality images were removed manually, and we were left with a total of 12,001 images for good- and poor-quality images to train the Inception-V1 algorithm using two quality groups at both ends of the spectrum. The complete list of scores and their quality map for good- and poor-quality are shown in Supplementary Table 5.

Finally, we divided the images into training, validation, and test groups. We allocated $70 \%$ of the images to the training group and the remaining $30 \%$ to the validation and test groups (Supplementary Table 3). The training, validation, and test sets (Supplementary Table 6) did not overlap.

\section{Algorithm architectures and training methods}

We employed a deep neural network (DNN) for embryo image analysis based on Google's Inception-V $1^{21}$ architecture, which offers an effective run-time and computational cost. ${ }^{44,45}$

Convolutional neural networks (CNNs) comprise several convolutions to pass the result to the next layer, pooling layers to combine the outputs of neurons into a single neuron, and fully connected layers, which represents the outputs. Inception-V1 architecture (popularly known as GoogLeNet) utilizes nine inception modules made of 22 layers with parameters and 27, including pooling layers. ${ }^{21}$ The preliminary three convolution nodes are combined with two max pooling operations and followed by nine inception modules that are stacked linearly. The architecture ends with a fully connected layer and then a softmax output layer to map the nonnormalized output to a probability distribution over predicted output classes. $^{21}$

To train this architecture, we used transfer learning, which involves training the whole network. We initialized our network parameters to the best parameter set that was achieved on ImageNet database. We then fine-tuned the parameters of all of the convolution filters of all outer layers of the network on our data (WCM-NY images) via back propagation. The training process was run for 50,000 iterations and implemented using the TensorFlow software library. ${ }^{46}$

\section{Evaluation of method and implementation details}

To implement our framework called STORK, we used Tensorflow version 1.4. $0^{46}$ and the TF-Slim Python library for defining, training, and evaluating models in TensorFlow. Training of our DNN method was performed on a server running the SMP Linux operating system. This server is powered by four NVIDIA GeForce GTX 1080 GPUS with 8 GB of memory for each GPU and 12 1.7-GHz Intel Xeon CPUs.

To evaluate the performance of our method, we used an accuracy measure, which is the fraction of correctly identified images. ${ }^{20}$ To assess the performance of different algorithms, precision-recall curves (PRCs) were used. Additionally, receiver operating characteristics (ROCs) were estimated. The ROC curve is depicted by plotting the true positive rate (TPR) versus the false positive rate (FPR) at various threshold settings. The accuracy is measured by the area under the ROC curve (AUC). ${ }^{47,48}$

\section{DATA AVAILABILITY}

The embryo imaging datasets analyzed in this study are not publicly available: due to reasonable privacy and security concerns, the embryo imaging data are not easily redistributable to researchers other than those engaged in the Institutional Review Board-approved research collaborations with the named medical centers. Our method is not specific to the datasets used in this study and users can train and test the deep learning model on any relevant imaging data.

\section{CODE AVAILABILITY}

The source code and the schematic guideline are publicly available at http://github. com/ih-lab/STORK. In addition, STORK is available through a web-based user interface at https://ivf-stork.org (password protected).

\section{ACKNOWLEDGEMENTS}

We acknowledge Dr. Fabien Campagne for useful discussions and providing additional computing resources for our analysis. This work was supported by start- up funds (Weill Cornell Medicine) to I.H. E.K. was supported by Swiss National Science Foundation under grant number 168574.

\section{AUTHOR CONTRIBUTIONS}

P.K., E.K., J.E.M., C.H., M.M., N.Z., O.E., and I.H. conceived the study. P.K., E.K., O.E., and I. $\mathrm{H}$. conceived the method and designed the algorithmic techniques. Q.Z., S.L., C.H., M. M., M.T., and N.Z. generated the datasets and prepared and labeled the images for various grades. P.K. and E.K. wrote the codes and performed computational analysis with input from O.E. and I.H. P.Z. and A.S. designed the STORK User Interface. Z.R. and L.C. provided critical reading and suggestions. P.K., E.K., Q.Z., O.E., N.Z., and I.H. wrote the paper, and all authors read, edited, and approved the final manuscript.

\section{ADDITIONAL INFORMATION}

Supplementary Information accompanies the paper on the npj Digital Medicine website (https://doi.org/10.1038/s41746-019-0096-y).

Competing interests: The authors declare no competing interests.

Publisher's note: Springer Nature remains neutral with regard to jurisdictional claims in published maps and institutional affiliations.

\section{REFERENCES}

1. Inhorn, M. C. \& Patrizio, P. Infertility around the globe: new thinking on gender, reproductive technologies and global movements in the 21 st century. Hum. Reprod. Update 21, 411-426 (2015).

2. Chandra, A., Copen, C. E. \& Stephen, E. H. Infertility and impaired fecundity in the United States 1982-2010: data from the National Survey of Family Growth. Natl. Health Stat. Report 67, 1-18 (2013).

3. Dyer, S. et al. International Committee for Monitoring Assisted Reproductive Technologies world report: Assisted reproductive technology 2008, 2009 and 2010. Hum. Reprod. 31, 1588-1609 (2016).

4. Manna, C., Nanni, L., Lumini, A. \& Pappalardo, S. Artificial intelligence techniques for embryo and oocyte classification. Reprod. Biomed. Online 26, 42-49 (2013).

5. Conaghan, J. et al. Improving embryo selection using a computer-automated time-lapse image analysis test plus day 3 morphology: results from a prospective multicenter trial. Fertil. Steril. 100, 412-419.e5 (2013).

6. Paternot, G., Debrock, S., De Neubourg, D., D'Hooghe, T. M. \& Spiessens, C. Semiautomated morphometric analysis of human embryos can reveal correlations between total embryo volume and clinical pregnancy. Hum. Reprod. 28, 627-633 (2013).

7. Tian, Y. et al. Predicting pregnancy rate following multiple embryo transfers using algorithms developed through static image analysis. Reprod. Biomed Online 34, 473-479 (2017).

8. Puga-Torres, T., Blum-Rojas, X. \& Blum-Narvaez, M. Blastocyst classification systems used in Latin America: is a consensus possible? JBRA Assist Reprod. 21, 222-229 (2017).

9. Saeedi, P., Yee, D., Au, J. \& Havelock, J. Automatic identification of human blastocyst components via texture. IEEE Trans. Biomed. Eng. 64, 2968-2978 (2017).

10. Taylor, T. H. et al. The origin, mechanisms, incidence and clinical consequences of chromosomal mosaicism in humans. Hum. Reprod. Update 20, 571-581, https:// doi.org/10.1093/humupd/dmu016 (2014).

11. Chen, M., Wei, S., Hu, J., Yuan, J. \& Liu, F. Does time-lapse imaging have favorable results for embryo incubation and selection compared with conventional methods in clinical in vitro fertilization? A meta-analysis and systematic review of randomized controlled trials. PLOS ONE 12, e0178720, https://doi.org/10.1371/ journal.pone.0178720 (2017).

12. Sundvall, L., Ingerslev, H. J., Breth Knudsen, U. \& Kirkegaard, K. Inter- and intraobserver variability of time-lapse annotations. Hum. Reprod. (Oxf., Engl.) 28, 3215-3221, https://doi.org/10.1093/humrep/det366 (2013).

13. Martinez-Granados, L. et al. Inter-laboratory agreement on embryo classification and clinical decision: Conventional morphological assessment vs. time lapse. PLOS ONE 12, e0183328 (2017).

14. Storr, A., Venetis, C. A., Cooke, S., Kilani, S. \& Ledger, W. Inter-observer and intraobserver agreement between embryologists during selection of a single Day 5 embryo for transfer: a multicenter study. Hum. Reprod. (Oxf., Engl.) 32, 307-314 (2017).

15. Rocha, J. C. et al. A method based on artificial intelligence to fully automatize the evaluation of bovine blastocyst images. Sci. Rep. 7, 7659 (2017).

16. Viswanath, P., Weiser, T., Chintala, P., Mandal, S. \& Dutta, R. Grading of mammalian cumulus oocyte complexes using machine learning for in vitro embryo 
culture. In Proc. of the IEEE-EMBS International Conf. on Biomedical and Health Informatics (BHI). 172-175, (IEEE, Las Vegas, NV, USA, 2016).

17. Santos Filho, E. et al. A method for semi-automatic grading of human blastocyst microscope images. Hum. Reprod. 27, 2641-2648 (2012).

18. Abramoff, M. D. et al. Improved automated detection of diabetic retinopathy on a publicly available dataset through integration of deep learning. Invest Ophthalmol. Vis. Sci. 57, 5200-5206 (2016).

19. Esteva, A. et al. Corrigendum: Dermatologist-level classification of skin cancer with deep neural networks. Nature 546, 686 (2017).

20. Khosravi, P., Kazemi, E., Imielinski, M., Elemento, O. \& Hajirasouliha, I. Deep convolutional neural networks enable discrimination of heterogeneous digital pathology images. EBioMedicine 27, 317-328 (2018).

21. Szegedy, C. et al. Going deeper with convolutions. In Proc. of the IEEE Conf. on Computer Vision and Pattern Recognition (CVPR). 1-9, (IEEE, Boston, MA, USA, 2015).

22. He, K., Zhang, X., Ren, S. \& Sun, J. Deep Residual Learning for Image Recognition. In Proc. of the IEEE Conf. on Computer Vision and Pattern Recognition (CVPR). 770-778, (IEEE, Las Vegas, NV, USA, 2016).

23. Litjens, G. et al. A survey on deep learning in medical image analysis. Med. Image Anal. 42, 60-88 (2017).

24. Esteva, A. et al. Dermatologist-level classification of skin cancer with deep neural networks. Nature 542, 115-118 (2017).

25. Gulshan, V. et al. Development and validation of a deep learning algorithm for detection of diabetic retinopathy in retinal fundus photographs. J. Am. Med. Assoc. 316, 2402-2410 (2016).

26. Saiz, I. C. et al. The Embryology Interest Group: updating ASEBIR's morphological scoring system for early embryos, morulae and blastocysts. Med. Reprod. Y. Embriología Clínica 5, 42-54 (2018).

27. Gardner, D. K., Lane, M., Stevens, J., Schlenker, T. \& Schoolcraft, W. B. Blastocyst score affects implantation and pregnancy outcome: towards a single blastocyst transfer. Fertil. Steril. 73, 1155-1158 (2000)

28. Arce, J. C. et al. Interobserver agreement and intraobserver reproducibility of embryo quality assessments. Hum. Reprod. 21, 2141-2148 (2006).

29. Subira, J. et al. Grade of the inner cell mass, but not trophectoderm, predicts live birth in fresh blastocyst single transfers. Hum. Fertil. (Camb.) 19, 254-261 (2016).

30. Irani, M. et al. Morphologic grading of euploid blastocysts influences implantation and ongoing pregnancy rates. Fertil. Steril. 107, 664-670 (2017).

31. Kinzer, D. R., Barrett, C. B., Penzias, A. S., Alper, M. M. \& Sakkas, D. Evaluation of a high implantation potential (hip) embryo grading system designed to reduce multiple pregnancy. J. Reprod. Health Med. 2, 11-16 (2016).

32. Yang, Z. et al. Selection of single blastocysts for fresh transfer via standard morphology assessment alone and with array CGH for good prognosis IVF patients: results from a randomized pilot study. Mol. Cytogenet. 5, 24 (2012).

33. Song, Y. Y. \& Lu, Y. Decision tree methods: applications for classification and prediction. Shanghai Arch. Psychiatry 27, 130-135 (2015).

34. Hebert, M., Collin-Vezina, D., Daigneault, I., Parent, N. \& Tremblay, C. Factors linked to outcomes in sexually abused girls: a regression tree analysis. Compr. Psychiatry 47, 443-455 (2006).

35. Kass, G. V. An exploratory technique for investigating large quantities of categorical data. Appl. Stat. 29, 119-127 (1980).
36. Ali, M. et al. Comparison of artificial neural network and decision tree algorithms used for predicting live weight at post weaning period from some biometrical characteristics in Harnai sheep. Pak. J. Zool. 47, 1579-1585 (2015).

37. Chen, W. et al. Establishing decision trees for predicting successful postpyloric nasoenteric tube placement in critically ill patients. JPEN J. Parenter. Enter. Nutr. 42, 132-138, https://doi.org/10.1177/0148607116667282 (2018).

38. Jeanray, N. et al. Phenotype classification of zebrafish embryos by supervised learning. PLOS ONE 10, e0116989 (2015).

39. Filho, E. S., Noble, J. A. \& Wells, D. A review on automatic analysis of human embryo microscope images. Open Biomed. Eng. J. 4, 170-177 (2010).

40. Matos, F. D., Rocha, J. C. \& Nogueira, M. F. A method using artificial neural networks to morphologically assess mouse blastocyst quality. J. Anim. Sci. Technol. 56, 15 (2014).

41. Segal, T. R. et al. Development of a decision tool to predict blastocyst formation Fertil. Steril. 109, 49-50 (2018)

42. Iwata, K. et al. Deep learning based on images of human embryos obtained from high-resolusion time-lapse cinematography for predicting good-quality embryos. Fertil. Steril. 110, 213 (2018).

43. Veeck, L. L. \& Zaninovic, N. An Atlas of Human Blastocysts, Vol. 286 (CRC Press, Taylor \& Francis, 2003)

44. Movshovitz-Attias, Y. et al. Ontological supervision for fine grained classification of Street View storefronts. In Proc. of the IEEE Conf. on Computer Vision and Pattern Recognition (CVPR). 1693-1702, (IEEE, Boston, MA, USA, 2015).

45. Schroff, F., Kalenichenko, D. \& Philbin, J. FaceNet: A unified embedding for face recognition and clustering. In Proc. of the IEEE Conf. on Computer Vision and Pattern Recognition (CVPR). 815-823, (IEEE, Boston, MA, USA, 2015).

46. Abadi, M. et al. TensorFlow: A system for large-scale machine learning. In Proc. of the 12th USENIX Symposium on Operating Systems Design and Implementation (OSDI). 265-283 (USENIX Association, Savannah, GA, USA, 2016).

47. Hanley, J. A. \& McNeil, B. J. The meaning and use of the area under a receiver operating characteristic (ROC) curve. Radiology 143, 29-36 (1982).

48. Zawistowski, M. et al. Corrected ROC analysis for misclassified binary outcomes. Stat. Med. 36, 2148-2160 (2017).

(c) (i)

Open Access This article is licensed under a Creative Commons Attribution 4.0 International License, which permits use, sharing, adaptation, distribution and reproduction in any medium or format, as long as you give appropriate credit to the original author(s) and the source, provide a link to the Creative Commons license, and indicate if changes were made. The images or other third party material in this article are included in the article's Creative Commons license, unless indicated otherwise in a credit line to the material. If material is not included in the article's Creative Commons license and your intended use is not permitted by statutory regulation or exceeds the permitted use, you will need to obtain permission directly from the copyright holder. To view a copy of this license, visit http://creativecommons. org/licenses/by/4.0/.

c) The Author(s) 2019 